13

\title{
Визуализация проводящих каналов в полимерных слоях методом атомно-силовой микроскопии с проводящим зондом
}

\author{
() В.М. Корнилов, ${ }^{1}$ А.Н. Лачинов, ${ }^{2}$ А.P. Юсупов ${ }^{1}$ \\ ${ }^{1}$ Башкирский государственный педагогический университет им. М. Акмуллы, \\ 450008 Уфра, Россия \\ ${ }^{2}$ Институт физики молекул и кристаллов Уфимского фредерального исследовательского центра РАН, \\ 450054 Уфра, Россия \\ e-mail: kornilov@anrb.ru
}

Поступило в Редакцию 29 марта 2021 г.

В окончательной редакции 29 марта 2021 г.

Принято к публикации 29 марта 2021 г.

\begin{abstract}
Представлены результаты экспериментального исследования локальных электрофизических свойств ультратонких полимерных пленок методами атомно-силовой микроскопии с проводящим зондом. Установлено, что визуализация мест протекания тока (проводящих каналов) возможна на участках, с которых механическим образом удален поверхностный слой. Проводящие каналы на токовом изображении имеют вид отдельных точек с высотой, соответствующей локально протекающему току. Установлено, что расположение наблюдаемых каналов хорошо соотносится с моделью проводимости по границам зерен надмолекулярной структуры полимера.
\end{abstract}

Ключевые слова: тонкие полимерные пленки, атомно-силовая микроскопия с проводящим зондом, проводящие каналы, надмолекулярная структура.

DOI: 10.21883/JTF.2021.10.51371.85-21

\section{Введение}

Развитие современной микро- и наноэлектроники во многом обусловлено потребностями современных информационных технологий, которые требуют разработки все более емких и быстродействующих устройств для записи и хранения информации с возможностью перезаписи и энергонезависимости. Наряду с традиционными типами запоминающих устройств (динамической, статической и флеш-памяти) большое внимание уделяется резистивной памяти, которая сочетает достоинства быстрой оперативной памяти с энергонезависимостью программируемой памяти. В основе этого типа памяти лежит структура металл-изолятор-металл, в которой может быть реализовано обратимое и резкое изменение проводимости диэлектрического слоя - эффект резистивного переключения. Описанные эффекты были обнаружены и исследованы в большом количестве классов соединений, включающих в себя неорганические [1-4] и полимерные слои субмикронной толщины $[5,6]$.

Анализ литературы показывает, что в литературе описывается только резистивное переключение (переход в высокопроводящее состояние), вызванное электрическим напряжением. В обзоре [7] представлены результаты исследования эффектов резистивного переключения в субмикронных пленках полигетероариленов, индуцированных не только электрическим полем, но и одноосным давлением, термоионизацией ловушек, электронным или ионным облучением, магнитным полем, изменением граничных условий на поверхности раздела металл-полимер. Очевидна перспективность использо- вания в электронике таких тонкопленочных элементов, электропроводящим состоянием которых можно управлять с помощью небольшого внешнего воздействия. При этом вопрос о механизме резистивного переключения в диэлектрических (полимерных) пленках напрямую связан, во-первых, с качеством и внутренней структурой пленки, а во-вторых, с инжекционно-контактными явлениями на интерфейсах электрод-полимер.

В работе [8] впервые был предложен механизм резистивного переключения, предполагающий образование проводящих шнуров (филаментов) в диэлектрическом слое. Шнуры могут рваться и переставать давать вклад в проводимость, при подходящих условиях шнуры могут образоваться вновь, что и обеспечивает основное свойство резистивного переключения: обратимый переход из низкопроводящего в высокопроводящее состояние. К настоящему времени предположение о шнуровании тока в процессе переключения является общепринятым практически для всех материалов, в которых обнаруживается резистивное переключение [9]. Исторически в литературе использовалось несколько терминов для обозначения проводящих участков в диэлектрическом слое: шнуры, нити, филаменты, каналы. В настоящей работе используется термин ,проводящие каналы“.

В связи с этим была поставлена задача экспериментального исследования локальных электрофизических свойств ультратонких диэлектрических полимерных пленок методом атомно-силовой микроскопии (АСМ) с проводящим зондом. Эта методика позволяет производить одновременное картографирование морфологии поверхности и регистрацию электрических неоднородностей в 
пленке полимера [10,11]. Предполагалось выяснить, существуют ли изначально в полимерном слое какие-либо особенности, благоприятствующие протеканию тока при резистивном переключении, или перенос заряда начинается в результате внешнего воздействия на полимер. Поэтому на данном этапе исследовалась полимерная пленка, не подвергавшаяся добавочному воздействию. Конечно, невозможно исключить факторы, которые обусловлены параметрами сканирования: силой контактного взаимодействия зонда с образцом и приложенным напряжением, их нужно учитывать при интерпретации результатов.

\section{1. Методика эксперимента}

Режимы исследования электрических параметров осуществлены в формате комплекса дополнительных программных и аппаратных средств, которые присоединяются к базовым системам сканирующего мультимикроскопа СММ-2000Т. В итоге одновременно с регистрируемой картой рельефа осуществляется поточечное снятие дополнительных карт, которые соответствуют данному рельефу. Дополнительные карты несут информацию об электрических потенциалах или об электрической проводимости с локальностью снятия данных до единиц ангстрем. Была реализована стандартная однопроходная методика сканирования топографии в контактной моде с одновременным измерением тока, протекающего через кантилевер. При проведении эксперимента были использованы АСМ-кантилеверы серии „GOLDEN“ CSG10 с проводящим покрытием PtIr, предназначенные для работы в контактном режиме, производства фирмы NT-MDT. Кантилевер устанавливался на АCM-столик микроскопа СММ-2000, имеющий электрическую развязку с корпусом прибора. Напряжение, которое подавалось на образец базовой системой микроскопа, управлялось из контрольной панели СТМ-режима в промежутке от -5 до $+5 \mathrm{~V}$ с точностью до $1 \mathrm{mV}$. В каждой точке скана измерялся ток, в соответствии с величиной которого формировалась токовая карта (т.е. изображение, которое получается одновременно с базовым АСМ-изображением).

Для исследования методом АСМ изготавливались полимерные пленки из полидифениленфталида (ПДФ) на полированных подложках из нержавеющей стали по методике, описанной в работе [12]. Толщина пленок задавалась концентрацией полимера в растворителе циклогексаноне. Для определения толщины пленок и выявления внутренней структуры в них создавалось углубление до поверхности подложки при помощи медного микрорезака. Стенка углубления представляла собой ступеньку с высотой, равной искомой толщине.

\section{2. Результаты исследования}

На первом этапе были получены изображения поверхности исходного образца, снятые одновременно в режиме АCM (поддержание постоянной силы) и в режиме регистрации тока, протекающего в месте контакта проводящего кантилевера с образцом. В результате многочисленных исследований было установлено, что в режиме поддержания постоянной силы в исследуемой области токовые особенности не регистрируются. Это связано с тем, что исходная полимерная пленка (образец) является сплошной и однородной по толщине (в данном эксперименте $115 \mathrm{~nm}$ ) и обладает хорошими диэлектрическими свойствами. Поэтому при работе в режиме поддержания постоянной силы взаимодействия кантилевера с образцом ток сквозь пленку не протекает и токовые особенности на карте протекания токов не регистрируются.

В связи с этим была предпринята попытка искусственно создавать деформированные участки полимерной пленки не на всю глубину. Это позволило бы получать изображение не поверхности, а внутренней структуры полимера. Свойства приповерхностной области полимерных слоев обычно отличаются от свойств полимера внутри слоя. В частности, известно, что боковые функциональные группы полимеров ориентируются преимущественно по нормали к поверхности раздела полимер-воздух. Предполагалось, что удаление приповерхностного слоя позволит пронаблюдать картину распределения протекающих токов в режиме постоянной силы взаимодействия кантилевера с образцом.

На рис. 1, $a$ представлен внешний вид деформированного участка полимерной пленки (морфология поверхности). Левая и нижняя части изображения соответствуют металлической подложке, которая стала видна после удаления части полимерной пленки в результате механической деформации. В правой верхней части виден участок полимерной пленки, оставшийся на поверхности подложки. Профиль сечения вдоль измерительной линии (видна на изображении) позволяет определить разницу высот между поверхностью подложки и поверхностью пленки, т.е. толщину пленки (в данном эксперименте $114 \mathrm{~nm}$ ). На рис. $1, b, c$ приведены изображения, полученные одновременно с увеличенного участка рис. $1, a$. Изображение на рис. $1, b$ характерно для внутренней (надмолекулярной) структуры полимерной пленки. Сoответствующий профиль сечения позволяет определить геометрические размеры структурных элементов внутри пленки. Рис. $1, c$ представляет собой карту распределения протекающего тока. Видно, что на токовом изображении кроме особенности, выглядящие на изображении как маленькие яркие точки. На геометрическом изображении (рис. $1, b$ ) таких точек не наблюдается, расположение точек коррелирует с границами элемента изображения, видного на обоих рисунках.

Изображение в токовом режиме представляет собой двумерное распределение величины протекающего тока 
$a$

Size: $[14.94 \mu \mathrm{m} \times 14.94 \mu \mathrm{m} \times 643.0 \mathrm{~nm}] \quad$ Size: $[2.183 \mu \mathrm{m} \times 2.183 \mu \mathrm{m} \times 187.7 \mathrm{~nm}]$ $(256 \times 256 \mathrm{pt})$
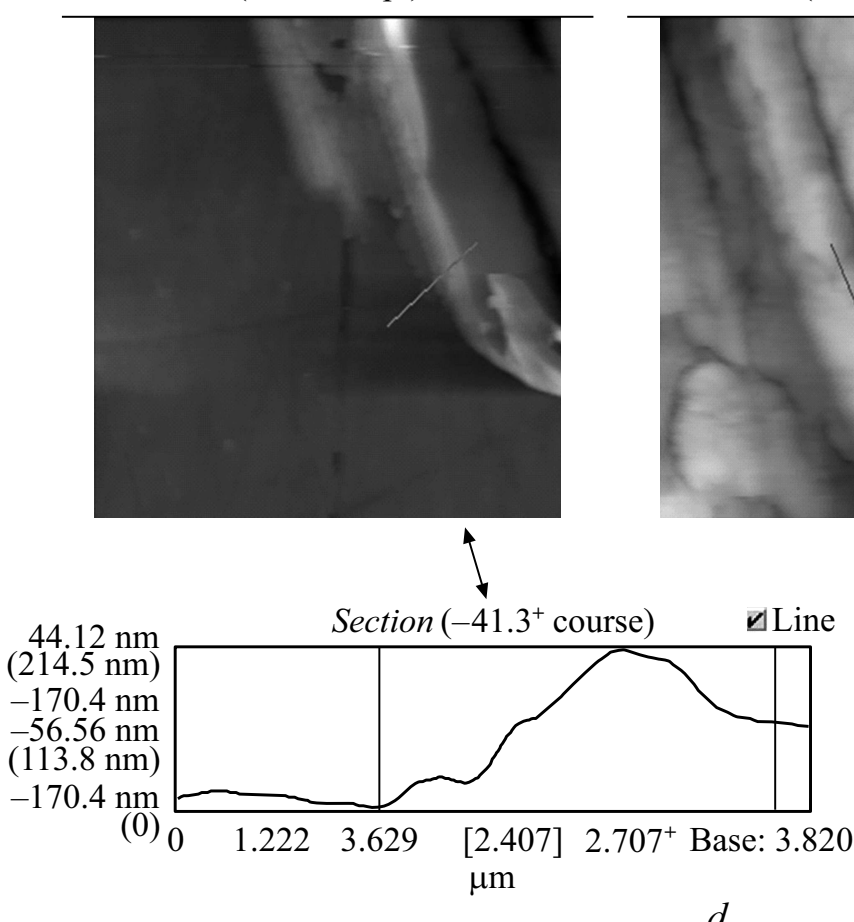

Size: $[1.205 \mu \mathrm{m} \times 1.205 \mu \mathrm{m} \times 17.36 \mathrm{nA}]$ $(330 \times 330 \mathrm{pt})$

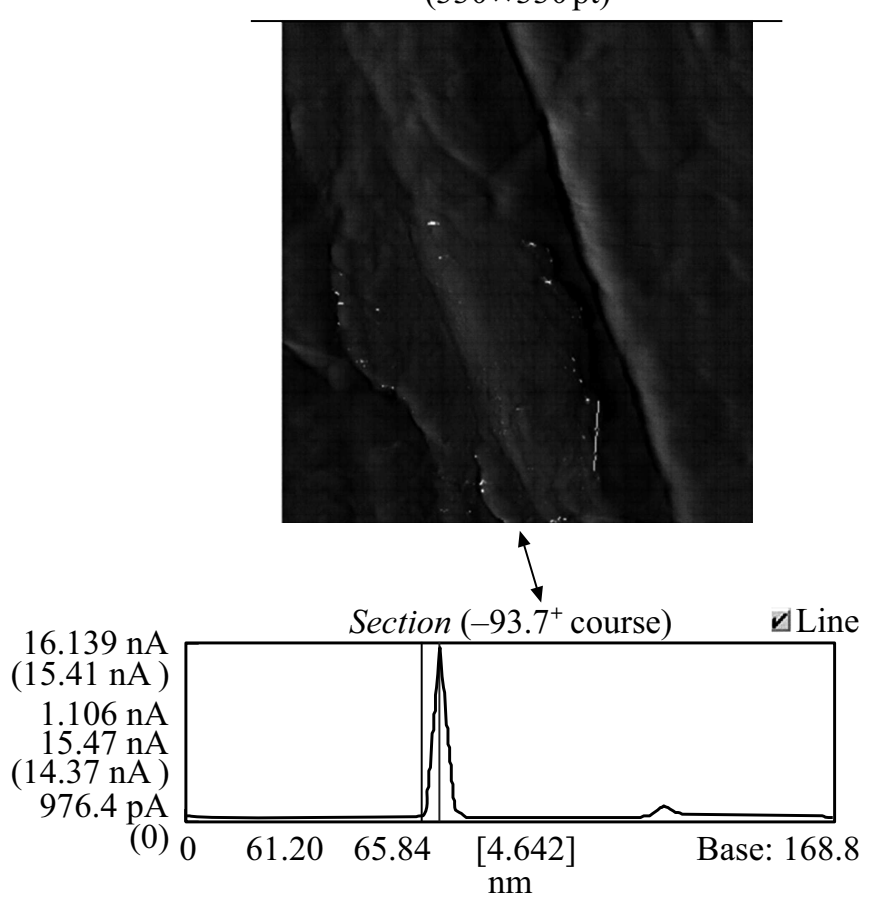

$b$

Size: $[2.183 \mu \mathrm{m} \times 2.183 \mu \mathrm{m} \times 81.37 \mathrm{nA}]$ $(299 \times 299 \mathrm{pt})$
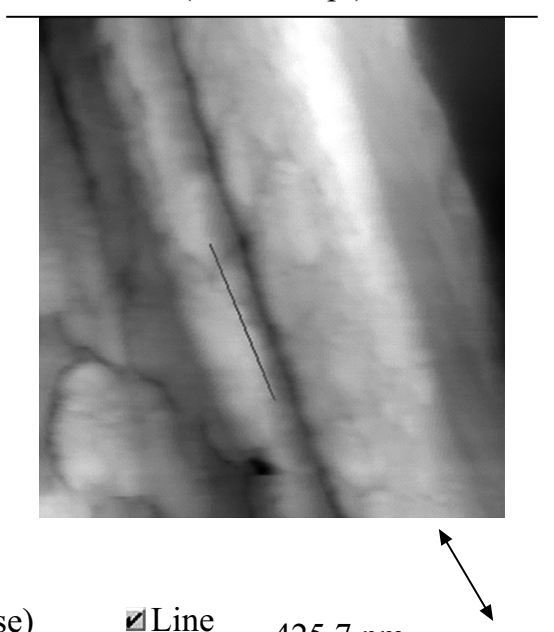

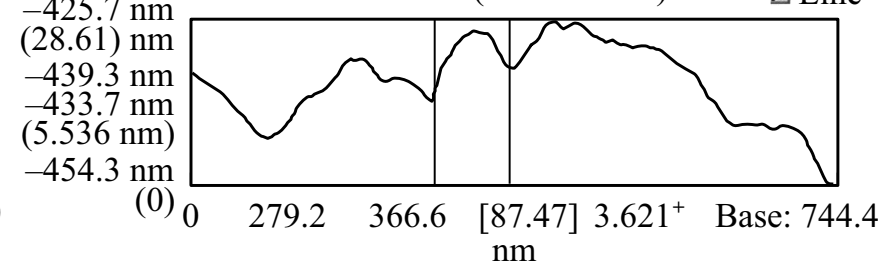

$e$

Size: $[273.7 \mathrm{~nm} \times 273.7 \mathrm{~nm} \times 280.8 \mathrm{nA}]$ $(330 \times 330 \mathrm{pt})$
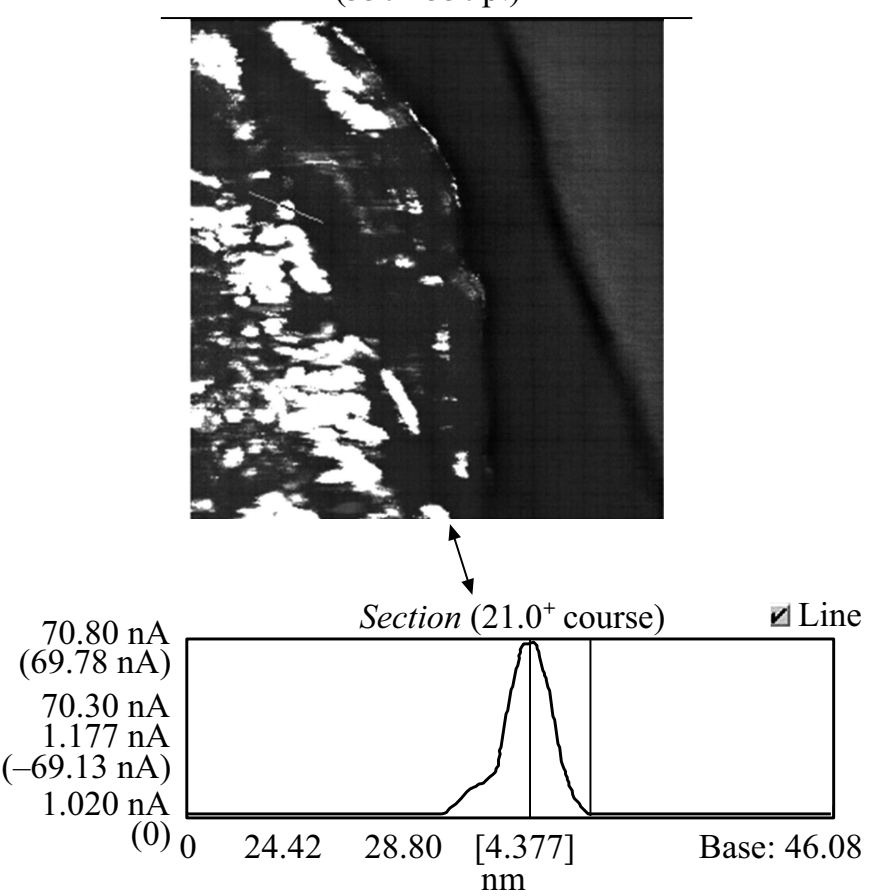

Рис. 1. Серия АСМ изображений деформированной полимерной пленки при разном масштабе увеличения: $a-$ общий вид деформированного участка и профиль сечения, толщина полимерной пленки $114 \mathrm{~nm} ; b-$ увеличенное изображение внутреннего строения полимерной пленки и соответствующий профиль, размер структурного элемента $87.5 \mathrm{~nm} ; c-$ изображение токопроводящих каналов на токовой карте, соответствующей топографии поверхности на рис. $1, b ; d-$ увеличенное изображение участка с проводящими каналами и профиль сечения; $e-$ следующая ступень увеличения изображения и профиль сечения. 
для всего массива исследованных точек поверхности. Токовые особенности на изображении имеют вид острых пиков, высота которых соответствует величине протекающего тока. На рис. $1, d$ представлено увеличенное изображение участка с проводящими каналами и профиль сечения. Величина тока в максимуме $14.37 \mathrm{nA}$, поперечник канала порядка $4.5 \mathrm{~nm}$. При многократном сканировании, особенно при увеличении масштаба изображения, наблюдается изменение величины токовых особенностей и формы их расположения (рис. $1, e$ ). По-видимому, это связано с деградацией полимерного слоя при многократном, хотя и слабом воздействии кантилевера. Величина тока, измеренная в одном из пиков, составила $69.13 \mathrm{nA}$, поперечник канала порядка $4.5 \mathrm{~nm}$.

\section{3. Обсуждение результатов}

Таким образом, было проведено экспериментальное исследование ультратонких пленок полимера на металле методом атомно-силовой микроскопии с проводящим зондом. АСМ-изображение является результатом совместного протекания нескольких процессов. Часть процессов задается аппаратно, и их можно регулировать (наличие или отсутствие обратной связи, величина силы взаимодействия кантилевера с образцом, величина напряжения на проводящем зонде, скорость сканирования и т.д.). Имеется также ряд факторов, влияющих на конечное изображение, которые обусловлены спецификой исследуемых объектов.

Было установлено, что толщина полимерной пленки порядка $115 \mathrm{~nm}$, а размер структурного элемента в деформированной области порядка $90 \mathrm{~nm}$ (рис. 1, $a, b$ ), что совпадает по порядку величины с результатами, полученными ранее [13]. Для того чтобы пронаблюдать токовые особенности внутри полимерного слоя в режиме поддержания постоянной силы взаимодействия, были искусственно созданы деформированные участки полимерной пленки не на всю толщину полимерной пленки, т.е. устранен поверхностный слой. В данном случае удалось зарегистрировать токовые особенности в виде ярких точек, расположенных приблизительно в пределах участка изображения (рис. 1,c-e). Расположение токовых особенностей (проводящих каналов) в основном сохраняется при изменении масштаба увеличения и при повторном сканировании. Величина тока, измеряемая в отдельном проводящем канале, заметно меняется, поперечные размеры каналов остаются в пределах 4-5 nm. При достижении больших масштабов увеличения измеряемая величина тока возрастает, но изображение становится неустойчивым и плохо воспроизводится. Это, по-видимому, связано с увеличением интенсивности взаимодействия кантилевера с образцом и деградацией полимерной поверхности.

При толщине полимерной пленки $115 \mathrm{~nm}$ и приложенном к острию кантилевера напряжении $5 \mathrm{~V}$ не происходит электрического пробоя пленки. Величины токов, протекающих сквозь отдельный канал, находятся в диапазоне от долей $\mathrm{nA}$ до десятков $\mathrm{nA}$. Приближенная оценка величины плотности тока, локально протекающего сквозь конкретный проводящий канал (изображение и профиль на рис. 1,c), дает значение порядка $10^{9} \mathrm{~A} / \mathrm{m}^{2}$. Электрическое сопротивление данного канала составляет $347 \mathrm{M} \Omega$, а удельное сопротивление $0.033 \Omega \mathrm{m}$. Отметим также, что токовые особенности расположены только в пределах участка поверхности размером порядка $0.8 \times 0.5 \mu \mathrm{m}$, причем их конфигурация и интенсивность не зависят от толщины полимерного слоя. Можно утверждать, что внутри полимерной пленки (после удаления поверхностного слоя) регистрируются участки субмикронного размера, в пределах которых наблюдаются проводящие каналы нанометровых размеров. При этом воздействие проводящего зонда микроскопа не приводит к возникновению новых каналов, граница между участком с проводящими каналами и окружающей диэлектрической матрицей четко прослеживается при увеличении масштаба изображения.

Поскольку измерения проводимости исследуемого образца проводились при нормальных условиях, наличие пленки воды на поверхности полимера и приложенное на острие напряжение существенно более $0.5 \mathrm{~V}$, могут приводить к локальным электрохимическим реакциям [14], которые, в свою очередь, могут быть одной из причин наблюдаемых вариаций измеренной карты протекания токов при повторном сканировании. Более точная оценка влияния электрохимических реакций с целью полного его устранения будет одной из задач в дальнейших экспериментальных исследованиях.

В соответствии с современной классификацией резистивное переключение может быть описано с точки зрения типа проводящего пути [9]. Первый тип соответствует нитевидному (филаментарному) проводящему пути, при котором резистивное переключение происходит в результате образования и разрыва проводящих нитей в изолирующей матрице. Второй тип проводящего пути соответствует механизму интерфейсного типа, при котором резистивное переключение происходит на границе раздела между металлическим электродом и диэлектриком. Этот механизм переключения обычно связан с резистивным переключением биполярного типа, наблюдаемым в полупроводниковых оксидах. Изменение конформации макромолекул также рассматривается как механизм переключения в полимерных резистивных запоминающих устройствах [6]. Некоторые несопряженные полимеры, содержащие карбазольные группы в боковой цепи, такие как производные поли( $\mathrm{N}$-винилкарбазола), могут проявлять электрически индуцированные конформационные изменения между случайной и региорегулярной структурой, что приводит к изменению состояния проводимости [15].

В любом случае формирование проводящих каналов обязательно обусловлено структурой и свойствами соответствующей диэлектрической (в нашем случае полимерной) матрицы. В работах $[12,13]$ было показано, что 


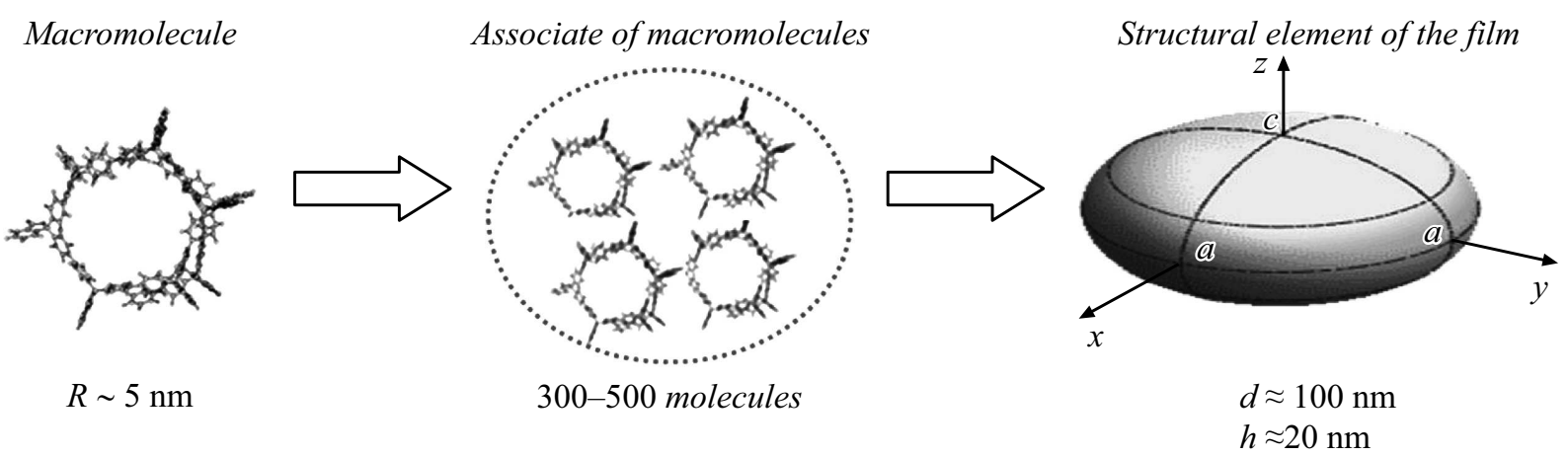

Рис. 2. Предполагаемый процесс формирования надмолекулярной структуры.

при гладкой и ровной внешней поверхности полимерная пленка ПДФ внутренне структурирована, причем внутреннее строение (надмолекулярная структура) пленок обусловлено ассоциацией макромолекул в исходном растворе. Ранее методами математического моделирования было установлено, что энергетически наиболее выгодными конфигурациями макромолекул ПДФ являются линейная синдиотактическая и спиральная изотактическая. Полимерные молекулы со спиральной структурой в исходном растворе объединяются в ассоциаты с преимущественной ориентацией боковых фрагментов на поверхности ассоциата. В частности, для полигетероариленов боковым является фталидный фрагмент, одна из связей которого может поляризоваться при захвате низкоэнергетического электрона.

На рис. 2 представлено схематическое изображение процесса формирования надмолекулярной структуры в пленке ПДФ.

При формировании пленки из раствора при быстром испарении растворителя ассоциатная структура фиксируется, причем границы между ассоциатами являются интерфейсами между полимерными поверхностями с преимущественной ориентацией дипольных моментов. Процессы, происходящие в таких структурах, в настоящее время активно исследуются в неорганических и органических кристаллах, а также на интерфейсах полимер-полимер. Границы раздела комплексных оксидов демонстрируют широкий спектр эффектов [16], таких как высокая подвижность носителей заряда и большие значения концентрации носителей, обнаружены также сверхпроводимость и гигантское магнетосопротивление. Как было выяснено ранее [17], многое в наблюдаемых эффектах определяется механизмами формирования интерфейсной области, включая последовательность чередования атомарных слоев и связанным с этим характером дипольного упорядочения вблизи границы раздела [18]. Модель „поляризационной катастрофы“, привлеченная для объяснения условий возникновения двумерного газа свободных электронов, оказалась достаточно правдоподобной и плодотворной [17]. В частности, были опубликованы работы, в которых сообщалось об обнаружении аналогичных эффектов вдоль границы раздела органических кристаллов [19] и аморфных полимерных пленок [20, 21].

В пределах интерфейса, существующего на границе раздела двух пленок ПДФ, образуется слой, обладающий аномально высокой проводимостью, которая возникает за счет двумерной электронной системы, сформированной слоем ориентированных на поверхности боковых фталидных групп с большим дипольным моментом. Определяющим механизмом в формировании проводящего интерфейса на границе раздела двух полимерных диэлектриков является поверхностная поляризация, обусловленная спонтанным дипольным упорядочением боковых фталидных групп. Сформированное этими диполями локальное поле формирует в области интерфейса благоприятные условия для переноса зарядов, о чем свидетельствуют большие значения подвижностей [22]. Перенос заряда по наноразмерным проводящим каналам реализуется, по-видимому, в соответствии с общими представлениями о шнуровании инжекционного тока [23]. Визуализация проводящих каналов является хорошей базой для детального исследования электрофизических свойств отдельного канала, что является предметом дальнейшей работы.

\section{Заключение}

В работе были получены новые экспериментальные данные о локальных электрофизических свойствах тонких полимерных слоев. Удалось визуализировать электропроводящие каналы нанометровых размеров в диэлектрической полимерной матрице. Методы АCM с проводящим зондом позволили определить линейные размеры проводящих каналов, величину и плотность тока в каналах.

Одновременное наблюдение мест протекания тока и надмолекулярной структуры полимера позволило использовать для описания эффекта модель формирования проводящего интерфейса на границе полимерных диэлектриков. Полученные данные важны для интерпретации результатов электрофизических измерений в субмикронных пленках полимера и позволяют целенаправленно влиять на состав и надмолекулярную структуру 
полимера при изучении свойств резистивного переключения.

\section{Благодарности}

Работа выполнялась при поддержке проекта „Зеркальные лаборатории“ Национального исследовательского университета „Высшая школа экономики“ и Башкирского государственного педагогического университета им. М. Акмуллы.

\section{Конфликт интересов}

Авторы заявляют, что у них нет конфликта интересов.

\section{Список литературы}

[1] D.S. Jeong, R. Thomas, R.S. Katiyar, J.F. Scott, H. Kohlstedt, A. Petraru, C.S. Hwang. Rep. Progr. Phys., 75, 076502 (2012). DOI: $10.1088 / 0034-4885 / 75 / 7 / 076502$

[2] J.S. Lee, S. Lee, T. Won. Noh. Appl. Phys. Rev., 2, 031303 (2015). dx.doi.org/10.1063/1.4929512

[3] S. Slesazeck, T. Mikolajick. Nanotechnology, 30, 352003 (2019). DOI: org/10.1088/1361-6528/ab2084

[4] M. Zhao, B. Gao, J. Tang, H. Qian, H. Wu. Appl. Phys. Rev., 7, 011301 (2020). DOI: 10.1063/1.5124915

[5] Q.-D. Ling, D.-J. Liaw, C. Zhu, D.S.H. Chan, E.T. Kang, K.G. Neoh. Progr. Polymer Sci., 33, 917 (2008). DOI: $10.1016 /$ j.progpolymsci.2008.08.001

[6] W.-P. Lin, S.-J. Liu, T. Gong, Q. Zhao, W. Huang. Adv. Mater., 26, 570 (2014). DOI: 10.1002/adma.201302637

[7] А.Н. Лачинов, Н.В. Воробьева. УФН, 176, 1249 (2006). DOI: 10.3367/UFNr.0176.200612a.1249 [A.N. Lachinov, N.V. Vorob'eva. Phys. Usp. 49, 1223 (2006). DOI: 10.1070/PU2006v049n12ABEH006176]

[8] G. Dearnaley, A.M. Stoneham, D.V. Morgan. Rep. Prog. Phys., 33, 1129 (1970).

[9] A. Sawa. Mater. Today, 11, 28 (2008). DOI: org/10.1016/S1369-7021(08)70119-6

[10] M. Trapatseli, D. Carta, A. Regoutz, A. Khiat, A. Serb, I. Gupta, T. Prodromakis. J. Phys. Chem. C, 119, 11958 (2015). DOI: 10.1021/acs.jpcc.5b01672

[11] D. Deleruyelle, C. Dumas, M. Carmona, C. Muller, S. Spiga, M. Fanciulli. Appl. Phys. Express, 4, 051101 (2011). DOI: 10.1143/APEX.4.051101

[12] Д.Д. Карамов, В.М. Корнилов, А.Н. Лачинов, В.А. Крайкин, И.А. Ионова. ЖТФ, 86, 124 (2016). [D.D. Karamov, V.M. Kornilov, A.N. Lachinov, V.A. Kraikin, I.A. Ionova. Tech. Phys., 61, 1085 (2016). DOI: org/10.1134/S106378421607015X]

[13] В.М. Корнилов, А.Н. Лачинов, Д.Д. Карамов, И.Р. Набиуллин, Ю.В. Кульвелис. ФТТ, 58, 1030 (2016). [V.M. Kornilov, A.N. Lachinov, D.D. Karamov, I.R. Nabiullin, Y.V. Kul'velis. Phys. Solid State, 58, 1065 (2016). DOI: $10.1134 / \mathrm{S} 1063783416050115]$

[14] Z. Shen, S. Hou, H. Sun, X. Zhao, Z. Xue. J. Phys. D: Appl. Phys., 37, 1357 (2004).

[15] S.L. Lim, Q. Ling, E.Y.H. Teo, C.X. Zhu, D.S.H. Chan, E.T. Kang, K.G. Neoh. Chem. Mater., 19, 5148 (2007). DOI: org/10.1021/cm071520x
[16] A. Ohtomo, H.Y. Hwang. Nature, 427, 423 (2004). DOI: org/10.1038/nature02308

[17] N. Nakagawa, H.Y. Hwang, D.A. Muller. Nature Mater., 5, 204 (2006). DOI: org/10.1038/nmat1569

[18] S. Thiel, G. Hammerl, A. Schmehl, C.W. Schneider, J. Mannhart. Sci., 313, 1942 (2006). DOI: $10.1126 /$ science.1131091

[19] J. Kirtley, J. Mannhart. Nature Mater., 7, 520 (2008). DOI: org/10.1038/nmat2211

[20] R.M. Gadiev, A.N. Lachinov, R.B. Salikhov, R.G. Rakhmeev, V.M. Kornilov, A.R. Yusupov. Appl. Phys. Lett., 98, 173305 (2011). DOI: 10.1063/1.3584135

[21] A.R. Yusupov, R.M. Gadiev, A.N. Lachinov, V.M. Kornilov, L.R. Kalimullina, A.F. Galiev, M. Kian, S.N. Salazkin. Synthetic Met., 274, 116733 (2021). DOI: org/10.1016/j.synthmet.2021.116733

[22] Р.М. Гадиев, А.Н. Лачинов, А.Ф. Галиев, Л.Р. Калимуллина, И.Р. Набиуллин. Письма в ЖЭТФ, 100, 276 (2014). DOI: doi.org/10.7868/S0370274X14160073. [R.M. Gadiev, A.N. Lachinov, A.F. Galiev, L.R. Kalimullina, I.R. Nabiullin. JETP Lett., 100, 251 (2014). DOI: org/10.1134/S0021364014160061]

[23] E. Tutiš, I. Batistic, D. Berner. Phys. Rev. B., 70, 161202(R) (2004). DOI: 10.1103/PhysRevB.70.161202 